\title{
Medical management of pediatric heart failure
}

\author{
Humera Ahmed, Christina VanderPluym \\ Department of Cardiology, Boston Children's Hospital, Harvard Medical School, Boston, Massachusetts, USA \\ Contributions: (I) Conception and design: None; (II) Administrative support: None; (III) Provision of study materials or patients: None; (IV) \\ Collection and assembly of data: None; (V) Data analysis and interpretation: None; (VI) Manuscript writing: All authors; (VII) Final approval of \\ manuscript: All authors. \\ Correspondence to: Christina VanderPluym, MD. Department of Cardiology, Boston Children's Hospital, 300 Longwood Avenue, Farley, 2nd Floor, \\ Boston, MA 02115, USA. Email: christina.vanderpluym@cardio.chboston.org.
}

\begin{abstract}
Pediatric heart failure is a complex, heterogenous syndrome that occurs relatively rarely in children, but carries a high burden of morbidity and mortality. This article reflects on the current state of medical therapy for both acute and chronic pediatric heart failure, based on expert consensus guidelines, and the extrapolation of data from trials performed in adults. For the management of acute heart failure specifically, we rely on an initial assessment of the perfusion and volume status of a patient, to guide medical therapy. This paradigm was adapted from adult studies that demonstrated increased morbidity and mortality in heart failure patients whose hemodynamics or examination findings were consistent with a PCWP $>18 \mathrm{mmHg}$ and a $\mathrm{CI} \leq 2.2 \mathrm{~L} / \mathrm{min} / \mathrm{m}^{2}$. The cornerstone of treatment in the acute setting therefore relies on achieving a euvolemic state with adequate cardiac output. In the chronic setting, patients are typically maintained on a regimen of an angiotensin converting enzyme inhibitor, a beta-blocker, and spironolactone. For those with refractory heart failure, intravenous milrinone therapy has become a mainstay of bridging children to cardiac transplantation. The pediatric-specific data driving these clinical practices are limited and often times, conflicting. The future of pediatric heart failure depends on collaboration, quality improvement, and a commitment to pediatric-specific indications for new medical and device therapies.
\end{abstract}

Keywords: Pediatrics; heart failure; congenital heart disease (CHD); inotropes; diuretics

Submitted Mar 14, 2020. Accepted for publication May 28, 2020.

doi: $10.21037 / \mathrm{cdt}-20-358$

View this article at: http://dx.doi.org/10.21037/cdt-20-358

\section{Introduction}

Pediatric heart failure is a complex, clinical and pathophysiologic syndrome that encompasses a diverse population of patients with congenital heart disease (CHD), cardiomyopathy, infectious and inflammatory diseases, oncologic processes, metabolic syndromes, renal failure, and malnutrition $(1,2)$. Although the estimated incidence of heart failure is relatively low at 0.9-7.4 per 100,000 children, it is a disease that carries a high burden of morbidity and mortality, with an in-hospital mortality rate of $7-26 \%(3,4)$. In the modern era, infants account for the majority (64\%) of heart failure admissions in patients $\leq 18$ years of age (4). The primary cardiac diagnosis at the time of admission is CHD (69\%), followed by arrhythmias (12-15\%), cardiomyopathy (13-14\%), and myocarditis $(\sim 2 \%)$. Recent analyses have revealed that the primary presenting complaints are often respiratory and/or gastrointestinal symptoms that can mimic more common pediatric illnesses, leading to incorrect and/or late diagnoses $(1,2,5)$.

Given the relative rarity of heart failure in pediatrics, and the heterogeneity of this population, there has been a paucity of clinical trials that have been performed primarily in pediatric patients. As a result, clinical guidelines for the treatment of pediatric heart failure have historically been reliant on expert consensus, and the extrapolation of data from trials performed in adults $(4,6,7)$. This article reflects on the current state of medical therapy for both acute and chronic pediatric heart failure. 
Table 1 The initial approach to the "wet" pediatric patient with acute heart failure

The wet patient (capillary wedge pressure $>18 \mathrm{~mm}$ )

Diuretics

Class I (level of evidence C)

1. Loop diuretics

Inhibition of sodium and chloride reabsorption via the $\mathrm{Na}+/ \mathrm{K}+/ 2 \mathrm{Cl}$ - cotransporter in the thick ascending limb of the loop of Henle: renal excretion of sodium chloride, potassium, and free water

2. Thiazide diuretics

Inhibition of sodium reabsorption in the distal renal tubules: renal excretion of sodium, water, potassium, and hydrogen ions

3. Mineralocorticoid antagonists

Competitively inhibits the binding of aldosterone to its receptor in the distal renal tubule: excretion of sodium, and the sparing of potassium

\section{Metolazone}

Inhibits sodium reabsorption in both the proximal and distal regions of the renal tubule: renal excretion of sodium, water, and potassium

Insufficient pediatric data to recommend use

5. Vasopressin antagonists

Antagonize the vasopressin-2 channel in the renal collecting duct: reduces water absorption through aquaporin channels

This the classification is based on an assessment of the patient's volume status (wet vs. dry), whereby the former correlates with hemodynamic or examination findings consistent with a pulmonary capillary wedge pressure $>18 \mathrm{mmHg}$.

\section{Acute heart failure management}

The initial approach to a pediatric patient presenting in acute decompensated heart failure involves the classification of patients into one of four distinct hemodynamic profiles, based on an assessment of the patient's volume status (wet $v s$. dry) and perfusion (cold $v s$. warm; Tables 1,2) (1). This paradigm was adapted from adult studies that demonstrated increased morbidity and mortality in heart failure patients whose hemodynamics or examination findings were consistent with a PCWP $>18 \mathrm{mmHg}$ and a $\mathrm{CI} \leq 2.2 \mathrm{~L} / \mathrm{min} / \mathrm{m}^{2}$ (8). While there are no data to confirm this association in pediatrics, this categorization provides a clinically useful tool for guiding appropriate medical therapy.

\section{The wet patient}

According to the most recent ISHLT guidelines for the management of pediatric heart failure, there is a Class I (level of evidence $\mathrm{C}$ ) indication for the initiation of diuretics in patients presenting with fluid retention associated with ventricular dysfunction (2). Diuretics should be continued until a euvolemic state has been achieved.

\section{Loop diuretics}

Loops diuretics work by inhibiting sodium and chloride reabsorption via an effect on the $\mathrm{Na}+/ \mathrm{K}+/ 2 \mathrm{Cl}-$ cotransporter in the thick ascending limb of the loop of Henle, thereby promoting the renal excretion of sodium chloride, potassium, and free water. Given an extensive clinical experience and favorable safety profile, loop diuretics are commonly used as first line therapy for diuresis in pediatrics (9). However, there are minimal data on the appropriate dose or frequency of administration of loop diuretics in pediatrics. In addition, it is often difficult to quantify the effectiveness of decongestive therapies in pediatrics, due to unreliable abdominal and pulmonary exams in uncooperative children, imprecise quantification of urine output in diapers with a mix of urine and stool, and variability in methods of weighing young children. Furthermore, children may not develop jugular venous distention in the setting of increased systemic venous pressures, and this marker becomes completely unreliable in children who have undergone direct anastomosis of the superior vena cava(e) to the pulmonary arteries. Finally, there are no data on the interpretation and significance of standard biomarkers of heart failure, such as BNP and its 
Table 2 The initial approach to the "cold" pediatric patient with heart failure

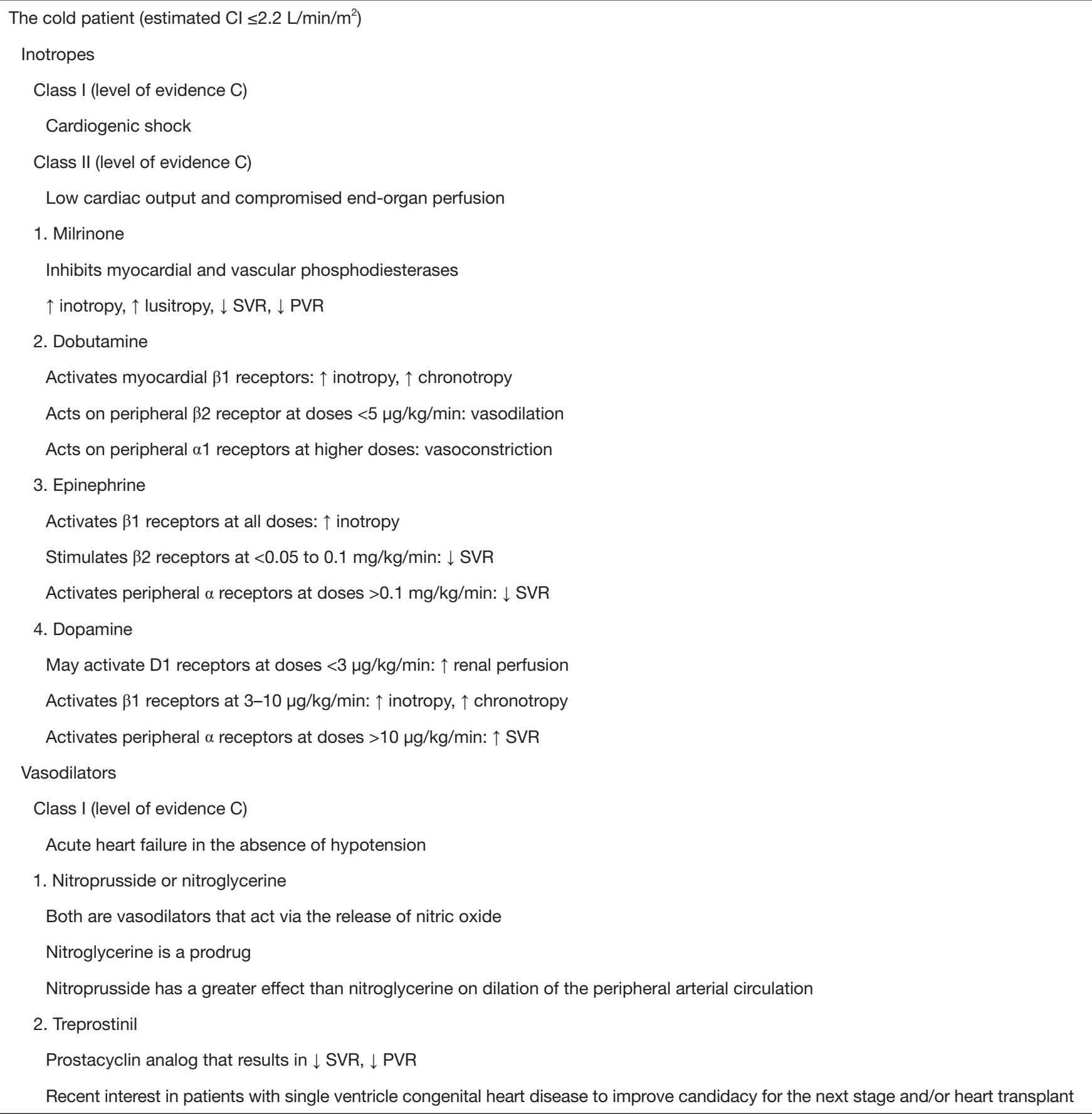

This the classification is based on an assessment of the patient's perfusion (cold vs. warm), whereby the former correlates with hemodynamic or examination findings consistent with a $\mathrm{Cl} \leq 2.2 \mathrm{~L} / \mathrm{min} / \mathrm{m}^{2}$.

precursor NT pro-BNP, in pediatric patients with single ventricle $\mathrm{CHD}$ and/or a systemic right ventricle.

These limitations carry important clinical implications, as inadequate decongestion - either due to diuretic resistance or inadequate dosing of diuretics—-has been associated with an increased risk of cardiovascular death or readmission in adults who are admitted for acute decompensated heart failure (10). Diuretic responsiveness (DR), defined as net fluid output 
or change in weight produced per 40 milligram furosemide equivalents, has been proposed as an alternative or adjunctive method of assessing the adequacy and effectiveness of a given decongestive strategy in adult patients. Price $e t$ al. explored the utility and significance of this concept in pediatrics, in a retrospective review of 108 consecutive patients $<21$ years old who were admitted to a single institution with a primary diagnosis of acute decompensated heart failure (11). The median cumulative dose of loop diuretic in the first 72 hours of admission was $85 \mathrm{mg}$ (IQR, 40-144 mg), resulting in a median net urine output of $513 \mathrm{~mL}$ (IQR, -182 to $1,523 \mathrm{~mL}$ ) in the same time period, and therefore a median DR of $6 \mathrm{~mL}$ net fluid output per milligram of loop diuretic. Patients were then categorized into categories of high and low DR, based on a response to diuretics that was above or below the median. In a multivariable analysis, low DR was independently associated with continuous renal replacement therapy, mechanical ventilation, and the composite primary outcome of mechanical circulatory support or death during the admission. These findings suggest that low DR may be an important early marker of illness severity, and should prompt an evaluation for underlying renal disease and/or low cardiac output that may require higher doses of diuretics, and/or inotropes and higher systemic blood pressures to maintain an adequate renal perfusion pressure (12).

This analysis did not comment on differences in DR for patients who received oral $v s$. intravenous diuretics. Given that gut edema and/or subclinical gut ischemia can be an important component of pediatric heart failureparticularly in patients with single ventricle CHDintravenous diuretics should be considered in patients with feeding intolerance, low DR, and/or significant fluid overload. Another important finding in this analysis was that $39 \%$ of patients received supplemental intravenous fluids comprising $>40 \%$ of total fluid intake, concurrent with diuretic use. While this was not associated with the primary outcome in this analysis, the concurrent use of intravenous fluids and diuretics has been associated with subsequent intensive care admission, mechanical ventilation, and continuous renal replacement therapy in adult heart failure patients (13).

In head-to-head comparisons of intermittent $v s$. continuous loop diuretics, there has been no reliable difference in urine output or mortality. There may, however, be some benefit to the use of continuous diuretics in neonates, particularly following cardiac bypass (14-16). In a prospective trial of 26 consecutive infants who had undergone congenital heart surgery and were randomized to either a continuous furosemide infusion of $0.1 \mathrm{mg} / \mathrm{kg} / \mathrm{hr}$ or intermittent bolus dosing of $1 \mathrm{mg} / \mathrm{kg}$ IV $\mathrm{q} 4 \mathrm{~h}$, the continuous infusion group had higher DR and a greater likelihood of achieving a negative fluid balance, despite a lower total daily dose of diuretic (17). The latter finding may have been secondary to fewer fluid shifts associated with the use of an infusion, ultimately resulting in a decreased need for volume resuscitation. A continuous infusion also decreases the necessity for central line access, which may theoretically decrease the risk of central lineassociated blood stream infections.

Other dosing and administration considerations include prematurity, which is associated with delayed clearance and a longer half-life of loop diuretics, necessitating decreased frequency of dosing (18). In addition, the chronic use of loop diuretics is known to induce tolerance, secondary to hypertrophy of the distal renal tubules, resulting in increased sodium reabsorption $(12,19)$. This can often be overcome by using a higher dose of the same diuretic; converting to a more potent loop diuretic, such as bumetanide; or using combination diuretic therapy (20). This strategy is also useful in patients with impaired renal function, as there is a strong correlation between renal excretion of furosemide and effective urine output (15).

\section{Thiazide diuretics}

Thiazide diuretics act by inhibiting the reabsorption of sodium in the distal renal tubules, which results in urinary excretion of sodium, water, potassium, and hydrogen ions. They are often administered 30 minutes prior to a dose of loop diuretic, in order to provide a synergistic effect, and/ or to overcome tolerance to loop diuretics. While there are limited pediatric data on the use of thiazides, the use of low dose thiazides (mean dose of $24 \mathrm{mg}$ /day hydrochlorothiazide equivalent) as a first-line agent for hypertension has been shown to reduce morbidity and mortality in adults (21).

\section{Mineralocorticoid antagonists}

According to the most recent ISHLT guidelines for the management of pediatric heart failure, there is a Class I (level of evidence C) indication for the initiation of mineralocorticoid antagonists in patients presenting with ventricular dysfunction. Spironolactone is the most commonly used mineralocorticoid antagonist in pediatrics. It is a synthetic steroid that competitively inhibits the binding of aldosterone to its receptor in the distal renal tubule, resulting in the excretion of sodium, and the sparing of potassium (22). The diuretic potency 
of spironolactone is quite limited, but its potassiumsparing effect has made it an important component of decongestion in the acute setting. This is particularly true in pediatrics, given the association of gastrointestinal symptoms, including vomiting, with the use of enteral potassium chloride supplements.

\section{Metolazone}

In patients with refractory fluid overload despite maximal doses of loop and thiazide diuretics, metolazone can be used to effectively augment diuresis in patients who can tolerate the enteral administration of medications. Metolazone is a potent, thiazide-like diuretic, that acts by blocking sodium reabsorption in both the proximal and distal regions of the renal tubule. Given a half-life of 12-24 hours, it is often dosed once or twice per day. Although there are some data to suggest its effectiveness in pediatric patients, its clinical use is limited by frequently associated electrolyte derangements $(23,24)$.

\section{Vasopressin antagonists}

These agents antagonize the vasopressin- 2 channel in the renal collecting duct to reduce water absorption through aquaporin channels. When used in combination with loop diuretics, they can provide symptomatic improvement in adults, but have not been shown to provide a survival benefit. A retrospective, single-center analysis of 25 infants who had undergone cardiac surgery and received tolvaptan for volume overload and poor DR to conventional diuretics, revealed that the use of tolvaptan was associated with increased DR in $68 \%$ of patients (25). Those patients who did respond had greater weight loss, and a shorter duration of intubation and length of ICU stay. Both responders and non-responders developed hypernatremia and increased serum osmolality, which ultimately recovered. There were 4 deaths in the patient cohort, the cause of which was not clearly delineated. While these data suggest the clinical utility of vasopressin antagonists for refractory volume overload in pediatrics, the $3 \mathrm{~T}$ (Comparison of Oral or Intravenous Thiazides vs. Tolvaptan in Diuretic Resistant Decompensated Heart Failure) trial did not reveal a difference in DR in a prospective, randomized, doubleblinded, double-dummy comparison of oral or intravenous thiazides $v s$. tolvaptan in adults hospitalized with hypervolemic acute heart failure and low responsiveness to loop diuretics (26). Given these equivocal findings and the limited data in pediatrics, there are insufficient data to recommend tolvaptan in pediatric heart failure (1).

\section{The cold child}

\section{Inotropes}

There is a Class I (level of evidence C) indication for the use of temporary intravenous inotropic support in pediatric patients presenting in cardiogenic shock, and a Class II (level of evidence C) indication) in patients with evidence of low cardiac output and compromised endorgan perfusion (2). However, given a high incidence of tachyphylaxis, and a risk of infection and arrhythmia associated with the use of inotropes, they should be used as a short-term strategy for stabilization and symptomatic relief. Concurrent with initiation, there should be a plan for escalation of therapy to mechanical circulatory support and/or cardiac transplantation if there have not been signs of meaningful clinical improvement (4). There is a Class IIa (level of evidence C) recommendation that while inotrope selection should be guided by an individual patient's clinical presentation, milrinone and/or dobutamine can be used as first-line rescue therapy, with epinephrine playing a role in the face of refractory hypotension and poor end-organ perfusion (2).

\section{Milrinone}

Milrinone inhibits myocardial and vascular phosphodiesterases, resulting in inotropy, lusitropy, and a decrease in systemic and pulmonary arterial resistances. Despite prospective, randomized trials that have revealed increased morbidity (hypotension and arrhythmias) and in-hospital mortality associated with the use of milrinone in adults with decompensated heart failure, intravenous milrinone plays a central role in the treatment of acute heart failure in pediatrics (27). Indeed, many large pediatric cardiology centers have established home milrinone programs that allow children with compensated heart failure to be discharged from the hospital with a continuous milrinone infusion via a peripherally inserted central catheter or a Broviac, so that they may await a heart transplant at home (28). Milrinone is commonly used in the post-operative period following the comprehensive Stage 1 to promote systemic blood flow and provide inotropic support to the single ventricle. It also plays an important role in decreasing the frequency of low cardiac output syndrome in the immediate post-operative period for children who have undergone cardiac bypass. Despite this rich clinical experience in pediatrics, the adult data would suggest that more rigorous studies are needed to evaluate the safety and efficacy of milrinone for the treatment of acute heart failure in children. The divergent clinical experience may be 
due to differing mechanisms of heart failure in children and adults (27). Because adults have more frequent coronary artery disease and myocardial scarring, they may be more sensitive to the increased myocardial oxygen demand associated with the use of milrinone and other inotropes.

\section{Dobutamine}

Dobutamine is a synthetic catecholamine that acts primarily on myocardial $\beta 1$ receptors, promoting increased inotropy and chronotropy. It also results in the opposing effects of vasodilation and vasoconstriction by acting on peripheral $\beta 2$ and $\alpha 1$ receptors, respectively. The former effect seems to be the dominant one at doses $<5 \mu \mathrm{g} / \mathrm{kg} / \mathrm{min}$, prompting caution when using dobutamine in patients with hypotension (29). It should also be used with caution in patients receiving beta blockers, as this may result in an unbalanced $\alpha 1$ effect, resulting in peripheral vasoconstriction (4). Dobutamine is used primarily for short-term, symptomatic improvement, as its onset of action is short. In a prospective study of 20 preterm neonates with myocardial dysfunction, the use of dobutamine resulted in an increase in stroke volume, cardiac output, and renal perfusion after 20 minutes of initiating the infusion (30).

\section{Epinephrine}

Epinephrine is a catecholamine that activates $\beta 1$-adrenergic receptors in the myocardium at all doses, and is thought to have a more powerful inotropic effect than dopamine. At doses $<0.05$ to $0.1 \mathrm{mg} / \mathrm{kg} / \mathrm{min}$, epinephrine also stimulates $\beta 2$-adrenergic receptors, resulting in reduced systemic vascular resistance. At doses $>0.1 \mathrm{mg} / \mathrm{kg} / \mathrm{min}$, it activates peripheral $\alpha$-adrenergic receptors, resulting in increased systemic vascular resistance.

\section{Dopamine}

Dopamine is a synthetic precursor to norepinephrine. When used at doses of $3-10 \mu \mathrm{g} / \mathrm{kg} / \mathrm{min}$, it activates $\beta 1$ adrenergic receptors in the myocardium, promoting increased inotropy and chronotropy that ultimately results in higher systemic blood pressures. At doses higher than $10 \mu \mathrm{g} / \mathrm{kg} / \mathrm{min}$, dopamine effects peripheral $\alpha$-adrenergic receptors, resulting in increased systemic vascular resistance, which can impede adequate cardiac output in states of cardiogenic shock. Finally, at doses $<3 \mu \mathrm{g} / \mathrm{kg} / \mathrm{min}$, dopamine is thought to activate D1 receptors, leading to renal arterial vasodilation, and improved renal perfusion (31). However, the only prospective, randomized trial to assess this effect, did not demonstrate an improvement in the glomerular filtration rate in adult patients with acute heart failure who received low dose dopamine infusions (32).

\section{Vasodilators}

For patients in acute heart failure with a significant myocardial volume load (e.g., valvar regurgitation), either nitroprusside or nitroglycerin has been found to significantly improve stroke volume and $\mathrm{CO}$ with a decrease in PCWP, without increasing myocardial oxygen demand and consumption. Given the risk of impairing adequate preload with the use of a vasodilator alone, the concurrent use of an inotropic agent is often required to maintain stroke volume and cardiac output (1).

\section{Special considerations for the patient with single ventricle CHD}

Patients with single ventricle CHD requiring aortic arch obstruction typically undergo a three-stage series of palliative open-heart surgeries, culminating in the Fontan (33). After the first few decades of life, the single ventricle inevitably develops depressed ventricular systolic function as a result of a combination of the strain that results from a morphologic right ventricle pumping to the systemic circulation, recurrent myocardial insults from cardiopulmonary bypass, inherent myopathy, and in some patients, chronic volume and pressures loads from valvar regurgitation and neo-aortic arch obstruction, respectively (34). For these patients, residual anatomic lesions should be corrected when possible. Decongestive and inotropic support should be used as with a two-ventricle circulation, with cautious use of vasodilators in patients with chronic hepatic congestion and dysfunction, who may have low SVR at baseline.

Fontan patients also inevitably develop a unique variant of acute-on-chronic heart failure related to failure of the Fontan circuit itself, as a physiological consequence of a chronic elevation in systemic venous pressures (35). There have been attempts to overcome this with early biventricular conversion and the development of a sub-pulmonary pump, but these efforts remain far from broad clinical adaptation $(36,37)$. In the interim, there may be a role for redefining "normal" pulmonary vascular resistance (PVR) and using intravenous pulmonary vasodilators in patients with single ventricle CHD, as a therapy for patients presenting with single ventricle failure, significant desaturation, and/ or venous hypertension. In a retrospective, single-center description of 17 single ventricle (2 shunt, 4 Glenn and 
3 Fontan) patients with an indexed PVR $>2.5$ iWU, hypoxia out of proportion to the stage of palliation, and/or decreased NYHA functional class, subcutaneous treprostinil was well tolerated, and resulted in decreased PVR, and improved oxygen saturation and exercise tolerance, in almost all patients (38). Six of the twelve shunt or Glenn patients achieved a sufficient decrease in PVR and improvement in symptoms, to proceed successfully through the next stage of palliation. Of the remaining six patients, three were bridged successfully to cardiac transplantation, and the remaining three remained on treprostinil at the time of publication, awaiting candidacy for the next stage or transplantation. Three of the six Fontan patients remained on stable therapy as outpatients, two had been weaned off, and one was successfully bridged to transplant.

\section{Chronic heart failure management}

\section{Angiotensin converting enzyme inbibitors}

For patients with left ventricular dysfunction, there is a Class 1 (level of evidence B) recommendation for the use of angiotensin converting enzyme inhibitors (ACEi) in symptomatic patients, and a Class IIa [level of evidence B] recommendation for asymptomatic patients (2). ACEis decrease adrenergic activity and the activation of the renin-angiotensin-aldosterone system, resulting in symptomatic improvement, reduced progression of heart failure, decreased hospitalization, and improved survival, in adults with heart failure (39-42). There have been no large randomized controlled trials of these medications in pediatric heart failure patients. In a retrospective review of 81 children with dilated cardiomyopathy and systolic ventricular dysfunction, those children who received an ACE inhibitor (primarily captopril in this study) had greater survival at 1 year of follow-up, and a trend toward improved survival at 2 years, when compared to patients who only received "conventional" therapy-consisting of digoxin, diuretics, and spironolactone (43).

Specifically for patients with Duchenne muscular dystrophy, there is a Class IIa (level of evidence B) recommendation for the use of ACE inhibitors; there is no recommendation for the optimal age of institution of therapy (2). In a prospective, randomized, doubleblind, placebo-controlled trial of perindopril in boys with Duchenne muscular dystrophy between the ages of 9.5 and 13 years with preserved ventricular function, there was a $27.4 \%$ absolute risk reduction in all-cause mortality at
10 years for patients in the perindopril group.

\section{Angiotensin receptor blockers (ARBs)}

Similar to its use in adults, ARBs are used primarily in children who are not tolerant of ACEis. In a prospective, randomized, placebo-controlled trial of 5,000 adults with heart failure, valsartan resulted in significant improvements in NYHA class, ejection fraction, signs and symptoms of heart failure, and quality of life as compared with placebo. The only randomized trial of ARBs in pediatrics compared lisinopril ( $5 \mathrm{mg} /$ day) and losartan $(25 \mathrm{mg} /$ day $)$ in 22 boys with Duchenne muscular dystrophy, revealing a significant improvement in left ventricular ejection fraction that was sustained at 1 year, without a difference between the two groups.

\section{Beta-blockers}

There is a Class IIa (level of evidence B) recommendation for the initiation of $\beta$-blockers in symptomatic children with systemic LV systolic dysfunction (2). Non-selective beta-blockers block the activation of $\beta$-adrenergic receptors located in the heart and kidneys ( $\beta 1)$; lungs, gastrointestinal tract, liver, uterus, vascular smooth muscle, and skeletal muscle ( $\beta 2)$; and fat cells $(\beta 3)$. The selective $\beta 1$-blockers are thought to promote long-term, positive myocardial remodeling by decreasing the amount and impact of catecholamines that are released chronically in heart failure, as a result of chronic baroreceptor stimulation and activation of adrenal medullary secretion (44). In addition, $\beta$-blockers decrease heart rate and blood pressure, resulting in decreased myocardial demand, improved coronary perfusion, improved cardiac output, and arrhythmia prevention.

While there is a clearly demonstrated survival benefit associated with the use of selective $\beta$-blockers in adults, the data are not as compelling in pediatrics. In a singlearm, prospective, multicenter trial of 20 children with cardiomyopathy or CHD, and stable moderate heart failure, patients treated with carvedilol demonstrated improved left ventricular ejection fraction and a trend toward improved survival (45). Interestingly, the control arm in this study was a similarly aged cohort of patients with dilated cardiomyopathy and stable, moderate heart failure, who were treated with standard oral heart failure therapy around the same time period, but had not been enrolled in the trial. This study did not control for placebo effects, or the known improvement in outcomes associated with enrollment in 
a clinical trial. The authors did note decreased benefit in single ventricle patients that was presumed to be secondary to an increased reliance on heart rate for sustained cardiac output, and a differing mechanism of heart failure.

In a multicenter, randomized, double-blind, trial of 161 children and adolescents with symptomatic systolic heart failure, there was no difference in outcomes when comparing high-dose carvedilol $(0.4 \mathrm{mg} / \mathrm{kg} / \mathrm{dose})$, lowdose carvedilol $(0.2 \mathrm{mg} / \mathrm{kg} / \mathrm{dose})$, and placebo (46). It was postulated that these results may have been due to underpowering of the study, inclusion of patients with a morphologic right ventricle, a large placebo effect, and inclusion of a high proportion of infants and toddlers. The latter point reflects differing pharmacodynamic and pharmacokinetic profiles when beta-blockers are used in children, which may necessitate higher doses relative to body weight, or more frequent administration, to achieve steady state $(47,48)$.

\section{Spironolactone}

There is a Class I (level of evidence C) recommendation for the use of aldosterone antagonists in children with systemic $\mathrm{LV}$ dysfunction, based on the strength of data from the adult heart failure guidelines. Spironolactone was studied in a prospective, randomized, double-blind, placebo-controlled study of 1,663 patients with severe heart failure and a left ventricular ejection fraction $<35 \%$, who were already being treated with an ACEi and loop diuretic +/- digoxin (49). The trial was terminated early due to a $30 \%$ risk reduction in the risk of death (both from progressive heart failure and sudden death from cardiac causes) in the spironolactone group. Patients treated with spironolactone also experienced a significant improvement in symptoms of heart failure, and had 35\% fewer hospitalizations. The incidence of serious hyperkalemia was minimal in both groups of patients.

\section{Digoxin}

There is a Class I (level of evidence C) recommendation against the use of digoxin in children with asymptomatic left ventricular dysfunction, given adult data that have shown no survival benefit in a similar adult population (50). For symptomatic patients, there is a class IIa (level of evidence $\mathrm{C}$ ) recommendation for its use at low levels (serum digoxin concentrations of $0.5-0.9 \mathrm{ng} / \mathrm{mL}$ ), with cautious use in patients with renal dysfunction, or in conjunction with drugs that can alter digoxin levels (e.g., carvedilol and amiodarone) (2). Digoxin works by reversibly inhibiting the Na-K ATPase, resulting in increased myocardial calcium stores and a subsequent increase in myocardial contractility, as well as lengthening of phases IV and 0 of the cardiac action potential and a subsequent decrease in heart rate. Given the physiologic benefit of these effects in heart failure, digoxin played a central role in the treatment of heart failure for many decades. More recently, the use of digoxin has fallen out of favor, following a number of reports that revealed equivocal or worse outcomes associated with the use of digoxin in the "modern era", i.e., in conjunction with modern beta-blockers and ACEis/ ARBs. These determinations were based on post-hoc analyses of major adult heart failure trials (e.g., MADITCRT, Val-HeFT) that revealed an increased frequency of arrhythmias and hypotension, and reduced left ventricular ejection fraction, in patients who were on digoxin at the time of enrollment in the study, when compared to those who were not $(51,52)$. These comparisons did not account for a potential difference in the severity of illness between the two groups. Because digoxin is commonly used as a second line agent for heart failure, those patients on digoxin at enrollment may have been sicker at baseline. Meta-analyses suggest a more favorable effect of the use of digoxin in heart failure; although the impact on survival remains unclear. One meta-analysis of 9 studies and 91,379 patients revealed that digoxin use was associated with a relative risk reduction of $14 \%$ for all-cause mortality (53). Another meta-analysis comparing digoxin and placebo in 52 studies and 621,845 patients, revealed no difference in all-cause mortality, but a relative risk reduction of $8 \%$ in all-cause hospital admissions, in patients who had been treated with digoxin (54).

There are minimal large-scale, prospective studies of digoxin use in pediatric heart failure. Recently, however, a number of retrospective, database studies have demonstrated a survival benefit associated with digoxin use in the interstage period for patients with SVCHD $(34,55,56)$. Due to the limitations of both retrospective and multicenter database studies, the mechanism of this survival benefit is unclear, but may be related to the inotropic and antiarrhythmic benefits of digoxin. Others have proposed that the benefit may stem from a reduction in heart rate, which allows for increased diastolic filling time, thereby increasing coronary perfusion (57). Although performing a prospective randomized controlled trial would be the gold standard to definitively evaluate the effect of digoxin in the interstage period, this may not be feasible, 
given the difficulty in obtaining an adequate sample size to detect differences. In addition, since publication of the aforementioned trials, there has been a practice shift at many centers toward the initiation of digoxin in the interstage period.

\section{Chronic outpatient inotropes}

At large pediatric cardiology centers, patients with stable, chronic heart failure may be discharged home on a single, continuous inotrope, while awaiting heart transplantation. The only large-scale analysis of this practice was a retrospective, intention-to-treat analysis of the UNOS database. Between 1999 and 2012, 106 patients with a median age of $10.1 \pm 6.4$ years were discharged home with a single inotrope, which was intravenous milrinone in $91 \%$ of cases (28). Of interest, $47 \%$ of patients had CHD- $80 \%$ of which was single ventricle CHD. The remainder of patients had dilated cardiomyopathy. Of the entire patient cohort, $85 \%$ of patients underwent transplantation successfully, and $8 \%$ were successfully weaned from support as outpatients. Death occurred in $6 \%$ of patients at a median of 40 days, secondary to progressive heart failure (3 patients), sudden cardiac death (1 patient), plastic bronchitis (1 patient), and influenza infection ( 1 patient). There were 5 (5\%) line infections and $1(1 \%)$ exit site/skin infection.

There are minimal data on the use of outpatient dopamine for chronic heart failure. In a single center series of seven patients with advanced heart failure who were discharged home with continuous inotropes, 3 patients received dopamine at $2-3 \mu \mathrm{g} / \mathrm{kg} / \mathrm{min}$, either alone or in combination with milrinone (58). There were no significant side effects or complications reported, and the use of dopamine was associated with an increase in ventricular ejection fraction, and a decrease in heart failure symptoms and hospital readmissions.

\section{Secondary causes of heart failure}

When evaluating the child with an initial presentation of heart failure, it is imperative to assess for secondary causes of heart failure, both to identify reversible causes of the presentation, and because heart failure may be refractory to treatment unless the underlying cause has been addressed. In the neonate presenting with heart failure, it is imperative to assess for underlying CHD, arrhythmias, infection, metabolic disorders, or mitochondrial disorders, of which heart failure may be a secondary presentation. Critical
CHD can present with heart failure symptoms in the first few days of life, as the patent ductus arteriosus closes. These include severe aortic stenosis, severe coarctation of the aorta (COA), obstructed total anomalous pulmonary venous return, transposition of the great arteries (TGA) with intact ventricular septum (IVS), and hypoplastic left heart syndrome. Critical CHD requires catheter-based and/or surgical intervention soon after the manifestation of symptoms, in order to prevent severe acidemia that can progress rapidly to worsening heart failure, organ dysfunction, and death. Children with left-to-right shunts typically present in the first $4-8$ weeks of life, as the PVR reaches its physiologic nadir, resulting in overcirculation. Those with large left-to-right shunts, including atrioventricular canal defects, aortopulmonary window, and large ventricular septal defects may present in the first two weeks of life. These children are managed with diuretics and progress to surgical repair once they have failed medical therapy, and before they develop irreversible changes to the pulmonary arteries, resulting in pulmonary hypertension.

All neonates presenting with heart failure should undergo evaluation of the coronary arteries to assess for primary coronary abnormalities, namely an anomalous coronary artery from the pulmonary artery (ALCAPA), as most children experience normalization of function after surgical reimplantation of the left coronary artery. All children should also undergo evaluation for evidence of myocarditis, both by assessing for a maternal history of infection during gestation, as well as obtaining inflammatory markers and viral PCRs in the infant. Finally, neonates should be started empirically on carnitine pending the results of metabolic labs to assess for inborn errors of metabolism.

In older children presenting with heart failure, in addition to assessing for CHD, cardiomyopathy, and myocarditis, attention should be paid to history or exam findings suggestive of renal failure, or a history of radiation or chemotherapy. Given the unreliability of the serum creatinine-based Schwartz equation in estimating the estimated glomerular filtration rate (eGFR) in children with chronic disease and/or malnutrition, a cystatin $\mathrm{C}$ should be obtained along with a basic metabolic panel, when possible. For children with profound renal injury, ultrafiltration or dialysis may be needed to treat volume overload that can ultimately progress to symptomatic heart failure. Standard heart failure therapies should be used with caution in patients with renal injury-ACE inhibitors can worsen existing renal injury, and spironolactone can aggravate the hyperkalemia that often results from renal dysfunction. 
Furthermore, milrinone can accumulate in children with renal injury, leading to hypotension that is difficult to overcome until the drug has been cleared.

In children undergoing active chemotherapy or radiation for various cancers, the chemotherapy protocol should be modified, if possible, to use less cardiotoxic doses or agents. For protocols that include hyperhydration, daily weights should be obtained, and children should be assessed for hepatomegaly or tachypnea that might indicate volume overload and the need for diuretics. In children with a remote oncologic history, standard heart failure therapies can be used, as detailed above, with close attention to organ dysfunction that might preclude the safe use of these agents.

\section{Future directions}

Given the heterogeneity and relatively rarity of pediatric heart failure, there is a growing movement among pediatric institutions to pool data and experiences, and to demand pediatric-specific indications for new drugs and devices. The Advanced Cardiac Therapies Improving Outcomes Network (ACTION) has emerged as a champion of efforts in North America to advance the understanding of heart failure in children and adults with CHD by promoting collaboration, data sharing, and the rapid dissemination of effective clinical practices (59). Created in 2018, it has grown to include over 44 centers across the United States and Canada. In the same period, a number of legislative initiatives, including the Pediatric Research Equity Act and the Best Pharmaceuticals for Children Act, have incentivized orphan disease research and pediatricspecific labeling at the time that a new product enters the market (60). As a result of these efforts, the evaluation and introduction of novel heart failure agents for children is underway.

The PANORAMA-HF study was a double-blind active comparator control trial of ENTRESTO ${ }^{\circledR}$ (valsartan/ sacubitril) versus enalapril. This study utilized a novel rank order heart failure end point to compare both groups after 52 weeks of follow-up. Over 200 of the planned 360 subjects had been enrolled when the Food and Drug Administration (FDA) preemptively approved Entresto for the treatment of heart failure due systolic dysfunction of a systemic $L V$ in children 1 year and older. This approval was based on an interim analysis of outcomes at 12 weeks, that showed a greater reduction of NT-proBNP in the Entresto arm (44\% reduction) than in the enalapril arm (33\% reduction) (61). While the difference between the groups was not statistically different, the reduction in NTproBNP observed in the pediatric population was equal to or greater than had been observed in the adult trial (62). Of importance, despite FDA approval for use in children, the manufacturers of Entresto (Novartis ${ }^{\circledR}$, Basel, Switzerland) are committed to completing the pediatric trial. This rapid approval of a therapy for the pediatric population highlights the FDA's commitment to expanding labelling for children, and ensuring post market surveillance of pharmaceutical and device therapies in children with heart failure.

Advancing the field of pediatric heart failure will require the concerted efforts of multiple centers, disciplines, regulatory bodies, and industries to identify, evaluate and apply novel therapeutics in this population.

\section{Acknowledgments}

Funding: None.

\section{Footnote}

Provenance and Peer Review: This article was commissioned by the Guest Editor (Roland Hetzer) for the series "Heart Failure in the Young and Old: Insights into Various Therapies" published in Cardiovascular Diagnosis and Therapy. The article has undergone external peer review.

Conflicts of Interest: The authors have completed the ICMJE uniform disclosure form (available at http://dx.doi. org/10.21037/cdt-20-358). The series "Heart Failure in the Young and Old: Insights into Various Therapies" was commissioned by the editorial office without any funding or sponsorship. The authors have no other conflicts of interest to declare.

Ethical Statement: The authors are accountable for all aspects of the work in ensuring that questions related to the accuracy or integrity of any part of the work are appropriately investigated and resolved.

Open Access Statement: This is an Open Access article distributed in accordance with the Creative Commons Attribution-NonCommercial-NoDerivs 4.0 International License (CC BY-NC-ND 4.0), which permits the noncommercial replication and distribution of the article with the strict proviso that no changes or edits are made and the original work is properly cited (including links to both the formal publication through the relevant DOI and the license). 
See: https://creativecommons.org/licenses/by-nc-nd/4.0/.

\section{References}

1. Kantor PF, Lougheed J, Dancea A, et al. Presentation, diagnosis, and medical management of heart failure in children: Canadian Cardiovascular Society guidelines. Can J Cardiol 2013;29:1535-52.

2. Kirk R, Dipchand AI, Rosenthal DN, et al. The International Society for Heart and Lung Transplantation Guidelines for the management of pediatric heart failure: Executive summary. J Heart Lung Transplant 2014;33:888-909.

3. Burstein DS, Shamszad P, Dai D, et al. Significant mortality, morbidity and resource utilization associated with advanced heart failure in congenital heart disease in children and young adults. Am Heart J 2019;209:9-19.

4. Rossano JW, Kim JJ, Decker JA, et al. Prevalence, morbidity, and mortality of heart failure-related hospitalizations in children in the United States: a population-based study. J Card Fail 2012;18:459-70.

5. Hollander SA, Addonizio LJ, Chin C, et al. Abdominal complaints as a common first presentation of heart failure in adolescents with dilated cardiomyopathy. Am J Emerg Med 2013;31:684-6.

6. Lipshultz SE, Sleeper LA, Towbin JA, et al. The incidence of pediatric cardiomyopathy in two regions of the United States. N Engl J Med 2003;348:1647-55.

7. Shaddy RE, George AT, Jaecklin T, et al. Systematic Literature Review on the Incidence and Prevalence of Heart Failure in Children and Adolescents. Pediatr Cardiol 2018;39:415-36.

8. Nohria A, Tsang SW, Fang JC, et al. Clinical assessment identifies hemodynamic profiles that predict outcomes in patients admitted with heart failure. J Am Coll Cardiol 2003;41:1797-804.

9. McCammond AN, Axelrod DM, Bailly DK, et al. Pediatric Cardiac Intensive Care Society 2014 Consensus Statement: Pharmacotherapies in Cardiac Critical Care Fluid Management. Pediatr Crit Care Med 2016;17:S35-48.

10. Neuberg GW, Miller AB, O'Connor CM, et al. Diuretic resistance predicts mortality in patients with advanced heart failure. Am Heart J 2002;144:31-8.

11. Price JF, Younan S, Cabrera AG, et al. Diuretic Responsiveness and Its Prognostic Significance in Children With Heart Failure. J Card Fail 2019;25:941-7.

12. Ter Maaten JM, Rao VS, Hanberg JS, et al. Renal tubular resistance is the primary driver for loop diuretic resistance in acute heart failure. Eur J Heart Fail 2017;19:1014-22.

13. Bikdeli B, Strait KM, Dharmarajan K, et al. Intravenous fluids in acute decompensated heart failure. JACC Heart Fail 2015;3:127-33.

14. Alqahtani F, Koulouridis I, Susantitaphong P, et al. A meta-analysis of continuous vs intermittent infusion of loop diuretics in hospitalized patients. J Crit Care 2014;29:10-7.

15. Schoemaker RC, van dDer Vorst MM, van Heel IR, et al. Development of an optimal furosemide infusion strategy in infants with modeling and simulation. Clin Pharmacol Ther 2002;72:383-90.

16. Wu MY, Chang NC, Su CL, et al. Loop diuretic strategies in patients with acute decompensated heart failure: a meta-analysis of randomized controlled trials. J Crit Care 2014;29:2-9.

17. Luciani GB, Nichani S, Chang AC, et al. Continuous versus intermittent furosemide infusion in critically ill infants after open heart operations. Ann Thorac Surg 1997;64:1133-9.

18. Pacifici GM. Clinical pharmacology of the loop diuretics furosemide and bumetanide in neonates and infants. Paediatr Drugs 2012;14:233-46.

19. Kim GJ, Capparelli E, Romanowski G, et al. Development of Tolerance to Chronic Intermittent Furosemide Therapy in Pediatric Patients. J Pediatr Pharmacol Ther 2017;22:394-8.

20. Jentzer JC, DeWald TA, Hernandez AF. Combination of loop diuretics with thiazide-type diuretics in heart failure. J Am Coll Cardiol 2010;56:1527-34.

21. Musini VM, Nazer M, Bassett K, et al. Blood pressurelowering efficacy of monotherapy with thiazide diuretics for primary hypertension. Cochrane Database Syst Rev 2014;(5):CD003824.

22. van der Vorst MM, Kist JE, van der Heijden AJ, et al. Diuretics in pediatrics : current knowledge and future prospects. Paediatr Drugs 2006;8:245-64.

23. Arnold WC. Efficacy of metolazone and furosemide in children with furosemide-resistant edema. Pediatrics 1984;74:872-5.

24. Segar JL, Robillard JE, Johnson KJ, et al. Addition of metolazone to overcome tolerance to furosemide in infants with bronchopulmonary dysplasia. J Pediatr 1992;120:966-73.

25. Kerling A, Toka O, Ruffer A, et al. First experience with Tolvaptan for the treatment of neonates and infants with capillary leak syndrome after cardiac surgery. BMC Pediatr 2019;19:57. 
26. Cox ZL, Hung R, Lenihan DJ, et al. Diuretic Strategies for Loop Diuretic Resistance in Acute Heart Failure: The 3T Trial. JACC Heart Fail 2020;8:157-68.

27. Felker GM, Benza RL, Chandler AB, et al. Heart failure etiology and response to milrinone in decompensated heart failure: results from the OPTIME-CHF study. J Am Coll Cardiol 2003;41:997-1003.

28. Birnbaum BF, Simpson KE, Boschert TA, et al. Intravenous home inotropic use is safe in pediatric patients awaiting transplantation. Circ Heart Fail 2015;8:64-70.

29. Bistola V, Arfaras-Melainis A, Polyzogopoulou E, et al. Inotropes in Acute Heart Failure: From Guidelines to Practical Use: Therapeutic Options and Clinical Practice. Card Fail Rev 2019;5:133-9.

30. Robel-Tillig E, Knupfer M, Pulzer F, et al. Cardiovascular impact of dobutamine in neonates with myocardial dysfunction. Early Hum Dev 2007;83:307-12.

31. Davis RF, Lappas DG, Kirklin JK, et al. Acute oliguria after cardiopulmonary bypass: renal functional improvement with low-dose dopamine infusion. Crit Care Med 1982;10:852-6.

32. Chen HH, Anstrom KJ, Givertz MM, et al. Low-dose dopamine or low-dose nesiritide in acute heart failure with renal dysfunction: the ROSE acute heart failure randomized trial. JAMA 2013;310:2533-43.

33. Ohye RG, Sleeper LA, Mahony L, et al. Comparison of shunt types in the Norwood procedure for single-ventricle lesions. N Engl J Med 2010;362:1980-92.

34. Ahmed H, Anderson JB, Bates KE, et al. Development of a validated risk score for interstage death or transplant after stage I palliation for single-ventricle heart disease. J Thorac Cardiovasc Surg 2020;160:1021-30.

35. Rychik J, Atz AM, Celermajer DS, et al. Evaluation and Management of the Child and Adult With Fontan Circulation: A Scientific Statement From the American Heart Association. Circulation 2019. doi: 10.1161/ CIR.0000000000000696.

36. Emani SM, McElhinney DB, Tworetzky W, et al. Staged left ventricular recruitment after single-ventricle palliation in patients with borderline left heart hypoplasia. J Am Coll Cardiol 2012;60:1966-74.

37. Rodefeld MD, Marsden A, Figliola R, et al. Cavopulmonary assist: Long-term reversal of the Fontan paradox. J Thorac Cardiovasc Surg 2019;158:1627-36.

38. Handler SS, Ogawa MT, Hopper RK, et al. Subcutaneous treprostinil in pediatric patients with failing singleventricle physiology. J Heart Lung Transplant 2017. doi: 10.1016/j.healun.2017.09.008.
39. Cohn JN, Johnson G, Ziesche S, et al. A comparison of enalapril with hydralazine-isosorbide dinitrate in the treatment of chronic congestive heart failure. N Engl J Med 1991;325:303-10.

40. Group CTS. Effects of enalapril on mortality in severe congestive heart failure. Results of the Cooperative North Scandinavian Enalapril Survival Study (CONSENSUS). N Engl J Med 1987;316:1429-35.

41. Investigators S, Yusuf S, Pitt B, et al. Effect of enalapril on survival in patients with reduced left ventricular ejection fractions and congestive heart failure. N Engl J Med 1991;325:293-302.

42. Packer M, Poole-Wilson PA, Armstrong PW, et al. Comparative effects of low and high doses of the angiotensin-converting enzyme inhibitor, lisinopril, on morbidity and mortality in chronic heart failure. ATLAS Study Group. Circulation 1999;100:2312-8.

43. Lewis $A B$, Chabot $M$. The effect of treatment with angiotensin-converting enzyme inhibitors on survival of pediatric patients with dilated cardiomyopathy. Pediatr Cardiol 1993;14:9-12.

44. Rossano JW, Cabrera AG, Jefferies JL, et al. Pediatric Cardiac Intensive Care Society 2014 Consensus Statement: Pharmacotherapies in Cardiac Critical Care Chronic Heart Failure. Pediatr Crit Care Med 2016;17:S20-34.

45. Blume ED, Canter CE, Spicer R, et al. Prospective singlearm protocol of carvedilol in children with ventricular dysfunction. Pediatr Cardiol 2006;27:336-42.

46. Shaddy RE, Boucek MM, Hsu DT, et al. Carvedilol for children and adolescents with heart failure: a randomized controlled trial. JAMA 2007;298:1171-9.

47. Albers S, Meibohm B, Mir TS, et al. Population pharmacokinetics and dose simulation of carvedilol in paediatric patients with congestive heart failure. Br J Clin Pharmacol 2008;65:511-22.

48. Laer S, Mir TS, Behn F, et al. Carvedilol therapy in pediatric patients with congestive heart failure: a study investigating clinical and pharmacokinetic parameters. Am Heart J 2002;143:916-22.

49. Pitt B, Zannad F, Remme WJ, et al. The effect of spironolactone on morbidity and mortality in patients with severe heart failure. Randomized Aldactone Evaluation Study Investigators. N Engl J Med 1999;341:709-17.

50. Digitalis Investigation G. The effect of digoxin on mortality and morbidity in patients with heart failure. $\mathrm{N}$ Engl J Med 1997;336:525-33.

51. Lee AY, Kutyifa V, Ruwald MH, et al. Digoxin therapy and associated clinical outcomes in the MADIT-CRT trial. 
Heart Rhythm 2015;12:2010-7.

52. Butler J, Anand IS, Kuskowski MA, et al. Digoxin use and heart failure outcomes: results from the Valsartan Heart Failure Trial (Val-HeFT). Congest Heart Fail 2010;16:191-5.

53. Vamos M, Erath JW, Hohnloser SH. Digoxin-associated mortality: a systematic review and meta-analysis of the literature. Eur Heart J 2015;36:1831-8.

54. Ziff OJ, Lane DA, Samra M, et al. Safety and efficacy of digoxin: systematic review and meta-analysis of observational and controlled trial data. BMJ 2015;351:h4451.

55. Oster ME, Kelleman M, McCracken C, et al. Association of Digoxin With Interstage Mortality: Results From the Pediatric Heart Network Single Ventricle Reconstruction Trial Public Use Dataset. J Am Heart Assoc 2016;5:e002566.

56. Brown DW, Mangeot C, Anderson JB, et al. Digoxin Use Is Associated With Reduced Interstage Mortality in Patients With No History of Arrhythmia After Stage I Palliation for Single Ventricle Heart Disease. J Am Heart Assoc 2016;5:e002376.

57. Van Hare GF. Perspective. Digoxin for interstage single ventricle patients: What could possibly go wrong?

Cite this article as: Ahmed H, VanderPluym C. Medical management of pediatric heart failure. Cardiovasc Diagn Ther 2021;11(1):323-335. doi: 10.21037/cdt-20-358
Congenit Heart Dis 2019;14:321-3.

58. Price JF, Towbin JA, Dreyer WJ, et al. Outpatient continuous parenteral inotropic therapy as bridge to transplantation in children with advanced heart failure. J Card Fail 2006;12:139-43.

59. Peng DM, Rosenthal DN, Zafar F, et al. Collaboration and new data in ACTION: a learning health care system to improve pediatric heart failure and ventricular assist device outcomes. Transl Pediatr 2019;8:349-55.

60. Bourgeois FT, Hwang TJ. The Pediatric Research Equity Act Moves Into Adolescence. JAMA 2017;317:259-60.

61. Shaddy R, Canter C, Halnon N, et al. Design for the sacubitril/valsartan (LCZ696) compared with enalapril study of pediatric patients with heart failure due to systemic left ventricle systolic dysfunction (PANORAMAHF study). Am Heart J 2017;193:23-34.

62. McMurray JJ, Packer M, Desai AS, et al. Dual angiotensin receptor and neprilysin inhibition as an alternative to angiotensin-converting enzyme inhibition in patients with chronic systolic heart failure: rationale for and design of the Prospective comparison of ARNI with ACEI to Determine Impact on Global Mortality and morbidity in Heart Failure trial (PARADIGM-HF). Eur J Heart Fail 2013;15:1062-73. 